\title{
La « pelle au cul » et autres joyeusetés langagières au temps du carnaval proscrit
}

The "pelle au cul » and other linguistic railleries during the time when Carnival was prohibited

Michel Biard et Jacques Guilhaumou

\section{Q OpenEdition}

\section{Journals}

\section{Édition électronique}

URL : https://journals.openedition.org/ahrf/11680

DOI : $10.4000 /$ ahrf. 11680

ISSN : 1952-403X

Éditeur:

Armand Colin, Société des études robespierristes

Édition imprimée

Date de publication : 1 septembre 2010

Pagination : 33-52

ISBN : 978-2-200-92633-5

ISSN : 0003-4436

\section{Référence électronique}

Michel Biard et Jacques Guilhaumou, « La « pelle au cul » et autres joyeusetés langagières au temps du carnaval proscrit », Annales historiques de la Révolution française [En ligne], 361 | juillet-septembre 2010, mis en ligne le 01 septembre 2013, consulté le 23 avril 2022. URL : http:// journals.openedition.org/ahrf/11680 ; DOI : https://doi.org/10.4000/ahrf.11680 


\title{
LA «PELLE AU CUL " ET AUTRES JOYEUSETÉS LANGAGIÈRES AU TEMPS DU CARNAVAL PROSCRIT
}

\author{
Michel BIARD \\ Jacques GUILHAUMOU
}

\begin{abstract}
Avec l'interdiction du Carnaval en 1791, toute une série de pamphlets modérés et royalistes s'empresse de décrire, dans la tradition burlesque, un monde à l'envers dans lequel le cul a tôt fait de se retrouver par-dessus tête, l'arme du ridicule pouvant alors se révéler cruelle en politique. Mais dans ce jeu qui mêle masque et cul, les hommes de plume "patriotes » ne sont pas en reste et n'hésitent pas, pour certains d'entre eux, à multiplier les occurrences du mot « cul » pour pimenter à souhait leur prose. Les Père Duchesne, que ce soit celui d'Hébert ou ceux de ses concurrents, aiment ainsi à décliner tant le mot que les situations burlesques pour mieux faire rire de leurs adversaires. Le langage de la farce grotesque, le « bel esprit », « la gaieté de l'esprit français » sont ainsi mobilisés dans une langue qui se veut « populaire » pour mieux atteindre ses cibles, tant les citoyens dont elle souhaite se faire entendre que des adversaires destinés à être d'abord ridiculisés, ensuite éliminés de la scène politique.
\end{abstract}

Mots-clés : Carnaval, langue populaire, corps, pamphlets, journaux, Père Duchesne.

(1) L'une des expressions favorites d'Hébert dans son Père Duchesne. Il rêve en effet de « foutre la pelle au cul » aux nobles et aux prêtres réfractaires, mais aussi, tour à tour, à « M. Pie » $\left(n^{\circ} 53\right.$ - mai 1791), aux « faux patriotes » $\left(n^{\circ} 59\right.$ - juin 1791), à « Louis le traître » $\left(n^{\circ} 64\right.$ - juillet $1791)$, à tous les « viédases » de l'état-major ( $\mathrm{n}^{\circ} 148$ - juin 1792), à l'Assemblée législative qualifiée de « [...] garce de législature, qui s'est prostituée $[. .$.$] » à tous les ennemis de la Révolution \left(\mathrm{n}^{\circ} 170\right.$ - septembre 1792)... bref, de la « foutre » à tous les « traîtres » $\left(n^{\circ} 204\right.$ - décembre 1792). 
Avant de repérer les emplois du mot «cul» dans le langage des années 1789-1792, grande est la tentation d'évoquer ce que l'un des plus grands cinéastes français actuels, Jean-Luc Godard, faisait dire à l'un de ses acteurs, de façon délibérément provocatrice : «Dans masculin, il y a masque et il y a cul $»^{2}$. Au moment du Carnaval, temps d'inversion des valeurs et de liberté de la parole, mais aussi de "cul par-dessus tête ", les masques peuvent de facto amener des culs sur le devant de la scène, sitôt que le haut et le bas se mêlent. D'ailleurs, le masque peut aussi dissimuler le cul, pour peu qu'on lui donne un aspect évocateur ou encore qu'on imagine une association entre le diable et Janus, l'odeur de soufre se mêlant alors à la confusion quant à la nature de ce qui doit être baisé lors du Sabbat ${ }^{3}$.

Dans son ouvrage sur L'œuvre de François Rabelais et la culture populaire au Moyen Âge et sous la Renaissance ${ }^{4}$, Mikhail Bakhtine qualifie le grotesque par contraste avec l'exagération. Dans le fait de mettre en évidence l'exagération, par exemple verbale, l'objectif demeure toujours d'attirer l'attention sur des phénomènes particuliers, hors normes, puis de les réduire à la norme analytique du moment, comme nous l'avons montré par ailleurs ${ }^{5}$. Nous en restons donc au niveau des représentations. Au contraire dans le grotesque, c'est la structure même de la vie qui est en cause : son figement dans un état de choses est nié au profit d'un « nouveau naissant $\gg$. Bakhtine prend ainsi l'exemple d'Arlequin donnant un coup de tête dans le ventre d'une femme, pour en libérer l'enfant qui y parle. Le grotesque se caractérise alors par une inversion de la topographie corporelle, le bas occupant le haut, ici la parole dans le ventre expulsée d'un coup de tête au moment même de la naissance de la vie. Et Bakhtine de préciser : « Le bas matériel et corporel corporalise et rabaisse les choses, mêle le corps au monde et renvoie à une conception particulière du bas corporel et de ses limites ». Ainsi, « le corps grotesque est un corps en mouvement, jamais prêt, ni achevé, toujours en état de construction, de création et lui-même construit un autre corps, il absorbe le monde et est

(2) On ne s'attardera pas ici sur le caractère pour le moins abrupt de la suite de cette citation (« dans féminin, il n'y a rien ». Jean-Luc Godard, Masculin-féminin, 1966).

(3) «Le cul du grand maître avait un visage derrière, \& c'est le visage de derrière qu'on baisait, \& non le cul » (Voyages imaginaires, romanesques, merveilleux, allégoriques, amusans, comiques et critiques, suivis des songes et visions, et des romans cabalistiques, Amsterdam et Paris, rue Serpente, 1789, tome 36, p. 343).

(4) Paris, Gallimard, 1970.

(5) Jacques Guilhaumou, «Modérer la langue politique à l'extrême. Les journalistes remarqueurs au début de la Révolution française ", $A H R F, \mathrm{n}^{\circ} 3,2009$, p. 21-46. 
absorbé par ce dernier ». On peut alors montrer en quoi le grotesque, et plus particulièrement le phénomène d'inversion qui s'y attache dans son lien étroit à « une nouvelle vie », concerne très directement le mouvement révolutionnaire.

C'est, de manière significative, avec le carnaval de 1791, interdit par la municipalité mais fêté par une série de pamphlets modérés et royalistes, en particulier un imprimé anonyme titré Le Carnaval de 1791. Avis au public, que l'on retrouve ce phénomène du grotesque. On y voit toute une série de personnalités politiques affublées de masques et de déguisements où la marque de l'inversion grotesque du haut et du bas est attestée. Ainsi, pour prendre quelques exemples, de Robespierre, avec un voile sur la tête, donc réduit à un fantôme; de Guillotin avec le masque de la mort, inversion de la vie; de Target en femme grosse, sa faconde d'avocat ainsi prise à revers de la naissance proche; d'Aiguillon, en tête de poissarde entourée de mouches, mal torché donc; etc. Un autre pamphlet anonyme, intitulé L'ombre du Mardi-Gras ou les Mascarades de la cour... pour suppléer au Carnaval de 1791 par un fou raisonnable, accentue le trait grotesque du « travestissement général », en l'assimilant à la révolution. Considérant que «la révolution s'est emparée des droits de Mardi-Gras » et qu'elle relève donc d'un « carnaval perpétuel », il s'agit, pour le pamphlétaire royaliste, de décrire l'attirail de la mascarade patriote, donc « le manteau populaire », pour arracher " le masque de l'imposture ». C'est alors qu'il s'évertue à « costumer les uns et les autres suivant les inclinaisons qui leur sont propres; en un mot, donner à chacun un habit à sa taille et décoré des emblèmes significatifs de ses passions, de ses vices et de ses vertus ». La scène la plus burlesque, avec son cortège d'inversion, est celle de la réception des « mascarades royales, nationales et épiscopales » à l'Hôtel de ville, donc auprès du maire Bailly. D'emblée, Bailly apparaît avec une queue de cheval en place de perruque, première inversion donc. Sur sa tête se trouvent également des cornes, signe de la bassesse. Le haut est bien ici le bas. Inversion du sexe également, déjà constaté dans le précédent pamphlet, avec le major-général Gouvion coiffé en femme. Quant aux officiers municipaux, l'un est affublé d'un bourrelet, désignant là encore la partie basse du corps, l'autre d'un broc de vin sur la tête, avec une note énigmatique au premier abord, « le tout pour rapprocher les naissances ", mais qui nous ramène à l'exemple cité ci-dessus d'Arlequin : ne s'agit-il pas de situer la parole des officiers municipaux dans un bas corporel qui, par la vertu du gonflement et du liquide, donnera naissance à une nouvelle vie? Nous sommes donc toujours dans un phénomène 
d'inversion grotesque, non seulement pris dans l'exhibition du bas à la place du haut, mais tout autant lié à la naissance, donc à la nouvelle vie négatrice de l'imposture et de la bassesse des personnalités politiques mises en scène.

Une autre sortie, plus inquiétante, de cette ambiance ainsi décrite de masques, de faux-semblants renvoie à l'action punitive du peuple, puisqu'il est aussi installé dans la salle du Conseil des cordes et poulies à l'adresse du peuple qui peut vouloir « finir cette comédie de carnaval par une tragédie à la 14 juillet ». Alors les occupants de l'hôtel de ville s'évertuent de remplir la tâche la plus démagogique qu'il soit : «Pour distraire la canaille et les affamés, on jettera dans la Place de Grève des petits pains et des cervelas, et on fera couler des fontaines de vin », toutes sortes de choses que l'on trouve à la foire soit en haut d'un mat de cocagne, soit en écoulement du haut vers le bas si l'on peut dire. Mais ici tout cela est abaissé. Une fois encore le haut passe vers le bas. Heureusement arrive en fin de parcours le Père Duchesne, le jour même de la cérémonie des cendres, qui «a préparé un paquet pour chacun ». Là encore le bas l'emporte sur le haut, mais au profit d'une nouvelle vie, de valeurs hautes : d'abord du monarque s'agenouillant devant « le célébrant en fumée », en l'occurrence le populaire marchand de fourneaux, puis de Marie-Antoinette, et ainsi de suite. De bordée en bordée lâchée, le Père Duchesne couvre donc ses pénitents royaux et leurs acolytes de sacs de cendres, leur barbouillant ainsi le crâne de manière à les sortir de leur " être nul», avec " un grand front vuide de sens » - il s'agit là encore de Bailly - pour qu'ils redeviennent «quelque chose », avec toujours l'appréhension que cet effort n'ira pas très loin, lorsqu'il les apostrophe en disant, « tu retourneras à rien »! Voilà donc le grotesque, et l'inversion du haut et du bas qui s'y associe, pris dans le principe que les hommes politiques du moment, face au peuple, ne sont rien, mais peuvent devenir quelque chose, au terme d'un rituel d'inversion dont le Père Duchesne se fait le porte-parole, le conciliateur. C'est d'ailleurs jusqu'au seuil de «la nation entière » que le Père Duchesne exerce son ministère salutaire, puisque, alors que « l'Assemblée nationale se présentera en corps, Mirabeau à leur tête », ce dernier se met à genoux devant le Père Duchesne et écoute sa semonce. Sa conclusion est claire : « Change de vie $»$ !

Mais ce carnaval des pamphlets est-il limité à des personnages fictifs fort réservés en Révolution, donc modérés et au-delà? Il n'en est rien. Ainsi du docteur Singulos dans ses Los Monolos-Dialogos ou Singularités du docteur singulos. Résultat authentique des efforts de sa 
Rhétorique sur tous les objets en ique concernant la chose publique ${ }^{6}$. Ce personnage insiste sur l'intérêt d'une "gaîté comique " au plus près de 《l'esprit patriotique $»^{7}$. Il s'agit alors de s'intéresser à des pamphlets « qui mordent sur tous les objets », à la fois « pour ou contre la Révolution », qui tiennent compte du « singulier» associant morale et badinage, donc qui ne contraignent pas le « caractère » par un « vouloir perpétuel », la dénonciation de la domination des Jacobins patriotes ${ }^{8}$. Il s'agit bien de « passer du bémol au diapason » en permanence, donc de ne pas «fixer le ton » de manière à ne point obliger, contraindre une opinion. Il s'agit aussi d'en finir avec « l'homme sans caractère » de « la classe des nullités », qui n'est rien donc. Enfin, il s'agit de «plaire par la variété et le piquant des tableaux ». C'est bien là ce que nous allons nous efforcer de faire dans la diversité même de la production pamphlétaire. Mais toujours en restant dans l'ordre de l'inversion burlesque tout en élargissant, si l'on peut dire, la descente du haut vers le bas, de la tête au ventre, avec l'accent sur le déplacement de la parole, à la recherche du caractère singulier de la Révolution, donc au mouvement du bas vers le haut, du « cul par-dessus tête ». Et c'est justement là où nous retrouvons notre « homme de caractère », le Père Duchesne, mais aussi d'autres héros de papier, au plus près de l'esprit républicain.

Le mot «cul » est présent depuis fort longtemps dans le langage, avec notamment tout l'apport de la verve rabelaisienne. On le retrouve en particulier dans l'expression populaire, fort usitée à Paris sous l'Ancien Régime, de « baiser le cul de la vieille » pour dire ordinairement que l'on perd au jeu sur toute la ligne ${ }^{9}$. Signalons aussi une autre locution adverbiale en la matière : « Le cul veut arriver avant la tête ». Toutes locutions donc qui se situent aussi dans un rituel d'inversion.

Si ce mot du « bas-langage $»^{10}$ est à proscrire pour ceux qui aiment à défendre dans leurs écrits un langage châtié ou/et à faire étalage de leur culture dans le cadre de cet art de la conversation si cher aux salons,

(6) Texte conservé à la BNF comme les précédents.

(7) Voir Antoine DE BAECQUE, Les éclats du rire. La culture des rieurs au XVIII ${ }^{e}$ siècle, Paris, Calmann-Lévy, 2000.

(8) Voir la série des Père Duchesne royaliste de la Place Saint-Michel reconstituée par Gérard Walter dans le Catalogue des journaux révolutionnaires, 1789-1799, Paris, Bibliothèque nationale, 1943.

(9) Voir par exemple dans le Dictionnaire comique, satyrique, critique, burlesque, libre et proverbial [...] de Philibert-Joseph Le Roux (1718).

(10) Dictionnaire du bas-langage, ou des manières de parler usitées parmi le peuple [...], Paris, d'Hautel et Schœll, 1808. 
d'aucuns aiment au contraire à l'employer afin de mieux transgresser, d'attirer l'attention en choquant, de mettre les rieurs de leur côté, mais aussi de séduire un lectorat populaire. Nul doute qu'Hébert, dans son Père Duchesne, a délibérément recours à ce qu'il nomme la langue « de la Sans-Culotterie $\gg{ }^{11}$ pour gagner et fidéliser un lectorat parmi ceux qui se reconnaissent dans un vocabulaire et des expressions que tout un chacun peut entendre quotidiennement dans l'espace public. De fort jolie façon, Jean Bart, autre héros fictif et parfois compagnon du Père Duchesne, baptise cela les « fleurs de rhétorique de mer $»^{12}$, formule qui ne manque pas d'avoir son équivalent sur la terre ferme. De nombreux autres auteurs manient, sinon le style du Père Duchesne, au moins le mot «cul » et les expressions qui en découlent, afin le plus souvent de ridiculiser des adversaires politiques pour mieux les combattre. À cet égard, la droite de l'Assemblée constituante est dès 1790 assimilée à un «cul-de-sac », ce qui permet de lui dénier d'emblée tout avenir ${ }^{13}$, de jouer ironiquement sur les couleurs à propos de ce « cul-de-sac des noirs $\gg{ }^{14}$, ou encore de se gausser de ses personnalités les plus en vue

(11) Je suis le véritable Père Duchesne, foutre!, $\mathrm{n}^{\circ} 257$, juillet 1793. Sur cette langue qu'il déclare être pour lui une "langue naturelle», voir Michel BIARD, Parlez-vous sans-culotte? Dictionnaire du Père Duchesne, 1790-1794, Paris, Tallandier, 2009 et Jacques Guilhaumou, La langue politique et la Révolution française, chapitre VI, édition numérique (2008) de l'édition de 1989, sur le site web Les classiques des sciences sociales (resp. Jean-Marie Tremblay).

(12) "Quand par habitude marine, il m'arrivera de lâcher un Foutre, ou quelqu'autre fleur de rhétorique de mer, mon imprimeur l'abrégera » (Je m'en $f$... ou Pensées de Jean Bart sur les affaires d'État, slnd [nº 3, mars 1790]. Cf. Ouzi Elyada, Presse populaire \& feuilles volantes de la Révolution à Paris 1789-1792. Inventaire méthodique et critique, Paris, Société des études robespierristes, 1991, p. 22).

(13) Le mot sert alors à désigner certaines adresses, notamment à Paris, mais donne aussi lieu à des usages visant à la dérision. En effet, s'il désigne comme aujourd'hui une rue n'ayant qu'une seule issue, il qualifie par ailleurs une affaire mal engagée et qui ne pourra aboutir. Ainsi, $L e$ Maréchal des logis des trois Ordres livre-t-il, en février 1789, toute une série d'adresses pour des personnalités de premier plan, dont plusieurs font référence à un cul-de-sac duquel ces hommes ne pourront s'extirper : celle du cardinal de Rohan est « cul-de-sac de Guéménée », celle de l'évêque de Sarlat « cul-de-sac de la Lâcheté », celle de l'abbé de Vermont « cul-de-sac de l'Empereur », celle du cardinal de la Rochefoucault « cul-de-sac des Patriarches ». Quant aux «journalistes », tous se trouvent logés au « cul-de-sac des Babillards », ce qui se passe de commentaire (cité dans Jean Pierre Louis de la Roche du MaINe, marquis de Luchet, Mémoires pour servir à l'histoire de l'année 1789, par une Société de Gens de Lettres, Paris, Lavillette, 1790, tome I, p. 117-121).

(14) Courier français, $\mathrm{n}^{\circ}$ du 15 août 1790; «Un journaliste, membre de l'assemblée nationale, pour faire entendre l'embarras où se jeta la minorité, qui avait épuisé tous les moyens de nuire à la constitution, donne au côté droit la désignation burlesque de cul-de-sac des noirs » (Pierre Jean Baptiste Nougaret, Anecdotes du règne de Louis XVI [...], Paris, sn, 1791, tome VI, p. 159); « Vainement les jean foutres du cul-de-sac des noirs ont rougi, vainement ils ont hurlé comme des loups enragés [...] » (Je suis le véritable Père Duchesne, foutre!, $\mathrm{n}^{\circ} 5$ - janvier 1791). 
tel l'abbé Maury qualifié de « Gargantua du cul-de-sac $»^{15}$. De la même manière, le mot «torche-cul» suffit à un journaliste pour disqualifier les feuilles de ses concurrents politiques. Ainsi, les Lettres bougrement patriotiques du véritable Père Duchêne, de Lemaire, expédient-elles en une ligne assassine le journal de Rosoi, La Gazette de Paris, très hostile à la Révolution (« Peut-on lire, sans se foutre en colère, l'exécrable torche-cul du brigand Durosoi $[\ldots]$ ? $\left.»^{16}\right)$, tandis qu'Hébert ne cesse de son côté de dénoncer ceux qui, à ses yeux, usurpent son titre et jusqu'au portrait de son marchand de fourneaux pour tromper les lecteurs («Après avoir cherché à me contre-faire de mille manières, des bougres de filous viennent encore d'ajouter à leur foutu torche-cu un portrait qu'ils assurent être le mien $»^{17}$ ). Reprenant semblable « propos torcheculatif » cher là encore à Rabelais, d'autres hommes de plume poussent l'irrespect jusqu'à appliquer l'image à des textes officiels, arrêtés municipaux ${ }^{18}$ ou décrets de l'Assemblée ${ }^{19}$, dès lors réduits à l'usage que tout bon sans-culotte, au propre (pour oser le mot) comme au figuré, doit faire des brefs du pape hostile à la Révolution (fig. 15). Et quand ce n'est pas le « torche-cul » qui est mentionné, le cul lui-même illustre la nullité de ce que l'on entend dénigrer, comme dans une pièce de théâtre intitulée La Journée des dupes, pièce tragi-politi-comique, représentée sur le théâtre national par les grands comédiens de la Patrie ${ }^{20}$. L'un des personnages, dont le nom donne lieu à un anagramme des plus transparents, Bimeaura y rencontre dans la rue des poissardes qui lui demandent, en parlant du droit de veto attribué au roi : « [...] dis-nous donc qu'euque c'est que cette varmine là? » Après avoir en vain tenté d'esquiver la

(15) La fille du Père Duchêne chassant à coups de balai l'abbé Mauri qui voulait coucher avec elle [...]. Cette feuille, rédigée par l'abbé Robin et publiée le 16 mars 1791, appartient au Père Duchesne de la place Henri IV, l'un des nombreux journaux concurrents de celui d'Hébert (cf. Ouzi Elyada, Presse populaire [...], op. cit., p. 140-146).

(16) Onzième lettre bougrement patriotiques du véritable Père Duchêne, de Lemaire, 4 novembre 1790 (cf. ibid., p. 62-82).

(17) Je suis le véritable Père Duchesne, foutre!, $\mathrm{n}^{\circ} 11$ - décembre 1790.

(18) Dans la Grande fureur du Père Duchêne contre la municipalité de Paris [...], de l'abbé Robin (cf. note 15), le héros populaire apostrophe ainsi Bailly, maire de Paris : «Quoi, jean-foutre, tu te plains sur tes torches-culs collés aux murailles, que l'anarchie règne toujours; mais c'est toi qui la causes » ( $\mathrm{n}^{\circ} 19$ bis, 18 mars 1791).

(19) «Comme j'étais content le jour où ils regardaient comme un torche-cul le décret de jean foutre, qui fut rendu [...]» (Je suis le véritable Père Duchesne, foutre!, nº 84 - octobre 1791).

(20) Sur cette pièce datée de 1790, cf. Michel BIARD, «Des violences populaires sur la scène théâtrale, ou l'impossible représentation (1789-1792) », dans Philippe BouRdin (dir.), La Révolution, 1789-1871. Écriture d'une Histoire immédiate, Clermont-Ferrand, Presses Universitaires Blaise-Pascal, 2008, p. 263. 
question (« Mais ce serait peut-être bien long à vous expliquer »), le fougueux tribun est contraint à exposer qu'il peut en exister de deux sortes, l'un absolu, l'autre suspensif. Et pour donner une image concrète du premier, il suggère aux poissardes d'imaginer que, au moment où elles sont en train de manger en famille, il prend soudain « [...] fantaisie au roi de dire veto, $\&$ sur le champ il prend votre soupe, $\&$ vous laisse-là, emportant votre dîner ». La réponse tombe aussitôt comme une sentence de la bouche d'une poissarde : «Qu'eu chienne de gueule! je ne voulons pas de cet absolu, ça rime à mon cul» (acte I, scène II). Rimer avec cul ou 《être fait comme mon cul $»^{21}$ renvoie à la même nullité et à des effets comiques similaires, tout comme les opposants à la Révolution aiment à se moquer des usages du vote en assemblée lorsque le suffrage se fait par assis-debout, au nom d'une transparence qui permet de désigner à l'opprobre public ceux qui refusent les décrets révolutionnaires. Organiser ainsi un scrutin signifie, selon eux, « décréter à cul levé $»^{22}$, autrement dit sinon privilégier le cul à la tête, à tout le moins « aller de cul et de tête » en un sens où le premier mot est là pour discréditer le second. La satire quant à cet autre retournement entre le haut et le bas n'est cependant pas l'apanage des hommes de plume hostiles à la Révolution, loin s'en faut, car le Carnaval marque avant tout le moment où les petits prennent la place des grands, et à ce jeu le cul et les expressions qu'il a fait naître sont assurés de se transformer en armes redoutables contre les puissants d'hier.

Pour les stigmatiser, quoi de mieux tout d'abord que l'évocation de l'Ancien Régime, dès lors qu'on en offre une vision caricaturale à souhait? À en croire journalistes et pamphlétaires, tout respirait alors la morgue, la servilité et la bassesse. Obnubilés à l'idée d'accéder un jour à la noblesse, les hommes les plus riches du tiers état ne faisaient en réalité que « péter plus haut que le cul $»^{23}$ et singer les manières des aristocrates. Pire, leur désir d'ascension sociale les poussait à une servilité dégradante, décrite par deux expressions presque synonymes, mais pourtant diffé-

(21) Anonyme, Voyage du Père Duchêne à Versailles, Paris, sd [1788].

(22) La Rocambole des journaux, $\mathrm{n}^{\circ} 12$ et 25, 9 février et 25 mars 1792. Ce journal, violemment hostile à la Révolution et qui se complaît dans un style comique, accuse également les « Jacoquins » de combiner leurs tactiques dans « un cul de taverne » ( $\mathrm{n}^{\circ} 11,5$ février 1792).

(23) « Je crois, dieu me pardonne, [...] que nous aurions mieux fait de ne pas péter plus haut que le cul et de vivre tout uniment en honnêtes-gens » (Je suis le véritable Père Duchesne, foutre!, $\mathrm{n}^{\circ} 193$-décembre 1792). 
rentes : «baiser le cul $»^{24}$ et « lécher le cul $»^{25}$. La première se veut écho du Sabbat dont les participants envoûtés par le Malin rendent hommage à celui-ci en lui « baisant le cul». Un personnage soupçonné de tenir un double langage, comme sait si bien faire le diable lorsqu'il prend une autre apparence, est alors souvent assimilé à Janus, tel Barnave, " monsieur double visage », et La Fayette; or ce dernier fait justement l'objet d'une adoration que ne renierait point Lucifer : «Quand on le voyait par ce côté-là, on était charmé, enchanté, et on l'aurait, sacré-dieu, volontiers baisé au cul $»^{26}$. Quant à la seconde expression, si le cul reste au centre de la délicate intention, elle atteste une soumission encore plus basse dans la mesure où, loin d'être une référence à une pratique sexuelle alors louée par toute une littérature soucieuse de vanter tout autant les charmes et plaisirs du «con» que du « cul», elle concerne des postérieurs que chacun doit imaginer d'un contact très désagréable, voire odieux. Comment imaginer autrement le fait de " [...] lécher le cul de ces foutus cafards d'inquisiteurs $\gg{ }^{27}$ ? Outre la crasse supposée du corps d'un inquisiteur de la sainte Église, nombre de lecteurs doivent avoir à l'esprit diverses références scatologiques, dont l'une des moins ragoûtantes est sans doute l'image de Sirop-au-cul, roi de comédie qui règne sur Merdenchine, entouré d'une Cour emplie de personnages aux noms évocateurs (Étronie son amante, Dégoutant, Merdencourt, Morvenbouche, Chiant-lit, etc.). L'hommage rendu à ce monarque fictif est en effet digne du Sabbat, puisque, à sa première apparition, il trône ${ }^{28}$ sur une chaise percée et « foire abondam-

(24) «Tandis que les moins forts s'imaginant être dans un prés fauchaient à droite et à gauche, et faisaient baiser la terre à tous ces jean foutres, qui autrefois leur auraient fait volontiers baiser leur cul » (ibid., $\mathrm{n}^{\circ} 40$ - mars 1791). Hébert se moque ici du rassemblement du camp de Jalès où des « contre-révolutionnaires » baisent ce qu'ils pensent être une terre à défendre, là où, avant la Révolution, ils allaient volontiers « baiser le cul» des nobles et des prêtres.

(25) Un autre verbe s'en rapproche : « flairer le cul». Le renvoi à «lécher» est alors complété de l'image du chien, grand habitué de cette pratique, voire du «limier »-chien et espion - au service d'un maître. Tel serait, selon Hébert, le cas de l'abbé Maury : «Depuis que tu as flairé le cul de nos ci-devant duchesses, \& servi de limier à nos évêques de cour; c'est foutu, ta pauvre $\&$ grosse tête a tant fermenté, que tu est devenu fol, \& que tu t'es persuadé qu'on te croirait un homme d'importance quand on te verrait louer tout ce que les autres désapprouvent » (ibid., $\mathrm{n}^{\circ} 7$ - novembre 1790).

(26) Le Dimanche gras du Père Duchêne ou Grand bal donné par le Père Duchêne au roi [...] (Le Père Duchesne de la place Henri IV, de l'abbé Robin, $\mathrm{n}^{\circ}$ du 6 mars 1791. Cf. supra note $15)$.

(27) « Nous foutrons le tour à ces bougres d'imbéciles là, \& nous les enverrons faire foutre en Espagne, en Italie \& lécher le cul de ces foutus cafards d'inquisiteurs » (Je suis le véritable Père Duchesne, foutre!, $\mathrm{n}^{\circ} 25$ - décembre 1790).

(28) Le verbe s'utilise déjà dans le sens figuré que notre langage a aujourd'hui conservé. 
ment » cependant que Dégoutant lui « baise le cul $»^{29}$. En 1789, nul n'oserait prétendre décrire de pareille manière les royales fesses, en revanche les «culs aristocrates" fournissent des cibles plus que tentantes pour qui veut ainsi mêler la servilité et l'ignoble, d'autant que les nobles sont réputés être eux-mêmes sortis d'un «cul» diabolique et par là même - nouvel écho du monde à l'envers - être ignobles (fig. 9, cahier couleur). Et, de foire en foire, toute une chaîne se trouve frappée d'ignominie, à l'exemple du club des Feuillants que le Père Duchesne tient pour être «[...] sorti du cul de la noblesse $»^{30}$ et non d'une simple scission du club des Jacobins. Le contact de ces êtres ignobles en devient si répugnant que le Père Duchesne préférerait encore le « sirop » du diable, lorsque proposition lui est faite de passer le Carnaval en compagnie de l'abbé Maury : « Mille noms d'une carogne, j'aimerai mieux, foutre, que le trou du cul de Lucifer me serve de bouteille $»^{31}$. En période de Carnaval, quand «tôt ou tard la tête emporte le cul $»^{32}$, la situation peut aussi se renverser, ainsi que le suggère de manière explicite l'un des nombreux pamphlets portant le nom du marchand de fourneaux : Le trou du cul du Père Duchesne, ou le Mouchoir des Aristocrates ${ }^{33}$.

Pour autant, si nobles et prêtres peuvent être ainsi tournés en ridicule, avec des attaques non dénuées de fondement, cela va de soi, il convient encore pour les révolutionnaires de dresser un tableau effrayant des dangers que représentent ces êtres ignobles. En effet, quand bien même le Carnaval, de manière éphémère, et la Révolution, que l'on espère durable, mettraient le monde à l'envers, tout patriote doit se persuader qu'un « aristocrate » est par nature incorrigible et ne cédera rien de ses privilèges sans se battre pour les préserver. Et si le cul leur passe un moment par-dessus tête, l'« aristocrate » et le roi en personne seront néanmoins, hélas, « [...] toujours comme ces petits pantins plombés que l'on pose sur la tête, et

(29) Charles François Racot DE GRANDVAL, Sirop-au-cul, ou l'Heureuse Délivrance. Tragédie hérö̈ merdifique, sl, Au Temple du goût, sd [1752].

(30) « Oui, foutre! c'est ainsi que ce club de pestiférés, est sorti du cul de la noblesse » ( $\mathrm{Je}$ suis le véritable Père Duchesne, foutre!, $\mathrm{n}^{\circ} 140$ - juin 1792).

(31) Le Grand Carnaval du Père Duchêne et son grand combat avec l'abbé Mauri [...] (Le Père Duchesne de la place Henri IV, de l'abbé Robin, $\mathrm{n}^{\circ}$ du 8 mars 1791. Cf. supra note 15).

(32) Ce proverbe populaire figure de nombreuses fois dans la bouche du Père Duchesne, dans la mesure où il autorise évidemment le rêve d'un monde à l'envers qui s'avérerait enfin pérenne : « Pour qu'un peuple soit heureux, foutre, il ne faut pas qu'il y ait des hommes trop riches et trop puissants, car tôt ou tard la tête emporte le cul » (Je suis le véritable Père Duchesne, foutre!, $\mathrm{n}^{\circ} 198$ - décembre 1792).

(33) Paris, Imprimerie de Chalon, 1790. 
qui retombent sur le cul $»^{34}$. Dès lors, et comme toujours en pareille situation, attiser les fantasmes du complot et de la trahison s'avère une arme redoutable. La hantise du complot s'exprime notamment à travers deux expressions : « remuer de tête et de cul »; « se tenir par le cul comme des hannetons ». Pour la seconde, l'affaire est vite entendue avec une sentence sans appel du Père Duchesne rendue contre « la bougre de calotte $\&$ la foutue aristracasserie qui se tiennent par le cul comme des hannetons [...] $»^{35}$. À l'exemple de toute autre " coterie», nobles et prêtres ne peuvent que communier dans l'hostilité à la Révolution et vouloir à tout prix sa mort afin de rétablir leurs pouvoirs et de faire revivre toutes les « tracasseries » de l'Ancien Régime. Ces dernières remettent alors en mémoire aux lecteurs du Père Duchesne tout ce dont la Révolution les a libérés (tailles et corvées, droits féodaux et seigneuriaux, dîmes, etc.) et, par conséquent, le souvenir noirci à souhait du régime défunt est supposé susciter un soutien quasi naturel à la Révolution en cours. Quant à la première expression, parfois employée également dans un sens favorable aux «patriotes» (mais, en ce cas, le verbe « remuer» tend à disparaître au profit de « aller de cul et de tête »), toutes les occasions sont bonnes pour la reprendre. S'agit-il de stigmatiser en 1791 le marquis de Bouillé, responsable de la répression qui s'est abattue l'été précédent sur la mutinerie des soldats suisses du régiment de Châteauvieux? La mutinerie devient le fruit d'une provocation organisée par ce même Bouillé pour mater les rebelles (« Le jean-foutre de Bouillé remuait de cul \& de tête pour faire soulever la garnison de Nancy $\left.[\ldots] \gg^{36}\right)$. Et lorsque, un an plus tard, ces Suisses condamnés au bagne sont libérés et accueillis triomphalement à Paris au cours d'une fête donnée en leur honneur par les Jacobins, la même expression sert à stigmatiser les mille et un complots supposés de Marie-Antoinette ( Je savais bien que Madame Veto remuerait de cul $\&$ de tête pour empêcher la fête que nous préparons aux braves soldats de Château-Vieux $\rangle^{37}$ ). À ceci près bien sûr que toute mention du « cul » de la reine ne manque pas de déclencher des critiques acerbes et des quolibets à caractère sexuel, nés à son sujet dès avant 1789. Ne sait-elle pas, cette nouvelle «Messaline », «tortiller du cul $\|^{38}$ et, si ses charmes ne suffisent point, acheter des soutiens grâce à l'argent mis à la disposition de son époux par la Liste civile? Parmi tous

(34) Je suis le véritable Père Duchesne, foutre!, nº 32 - février 1791.

(35) Ibid., n ${ }^{\circ} 25$ - décembre 1790.

(36) Ibid., no 60 - mars 1791.

(37) Ibid., n ${ }^{\circ} 122$ - avril 1792.

(38) Ibid., n ${ }^{\circ} 110$ - janvier 1792. 
ceux qui cèdent à ses attraits et à l'appât du gain, le marquis de La Fayette jouit d'une position privilégiée, car sa capacité à incarner Janus le prédispose à «tourner le cul» et à faire «tourner» celui des autres. Il arrive, certes, que lui-même soit trahi ( $« \mathrm{Tu}$ as cru séduire Mirabeau, qui a pris ton argent et t'a tourné le cul $»^{39}$ ), mais il n'en fait pas moins figure de grand manipulateur qui s'affiche en faveur du " peuple» tout en recevant des fonds secrets de la Cour. Si en 1790 le Père Duchesne d'Hébert a pu parfois participer aux louanges à son égard, le ton change vite. En septembre 1791, alors que la fusillade du Champ de Mars a ouvert des fractures irréversibles, contraint pour quelque temps les républicains au silence, et décillé bien des yeux quant au rôle du " héros des deux mondes », les conseils du marchand de fourneaux deviennent abrupts et récupèrent alors l'expression « tourner le cul » pour signifier l'abandon prochain du traître par le « peuple» : «Foutre, quand l'idole n'est plus, celui qui la veille ployait le genou, \& lui présentait l'encens; le lendemain lui tourne le cul, \& il est le premier à la fouler aux pieds $\rangle^{40}$. Désormais, le roi des Français lui-même n'est plus guère épargné, transformé en « roi de papier » ${ }^{41}$ livré à la féroce ironie et à l'imagination sans borne des hommes de plume, mais aussi des dessinateurs et graveurs. Varennes apparaît comme un épisode qui n'a trompé personne, en dépit des déclarations de l'Assemblée constituante sur le prétendu enlèvement du roi, et qui ne manquera pas d'être suivi d'autres tentatives de fuite. Relâcher la surveillance de la famille royale ne peut qu'aboutir à une nouvelle « évasion » et, à terme, au prix d'une autre inversion des mots, à l' " invasion » des troupes étrangères qui bousculeront les soldats français trahis par des officiers rêvant de saisir la première occasion pour « tourner le cul ». En août et septembre 1791, le Père Duchesne emploie une expression encore plus forte pour marquer la trahison de «Louis le faux » : "On dit partout que quand nous vous aurons donné la clef des champs, vous allez encore une fois nous brûler le cul, pour aller à la tête des Aristocrates et des Autrichiens $»^{42}$; «non, foutre, jamais on n'oubliera que malgré toutes ces belles promesses, le roi nous a brûlé le cul, pour s'aller jetter au milieu de nos ennemis $[\ldots] »^{43}$.

(39) Sermon bougrement patriotique du véritable Père Duchesne, à l'occasion de la nouvelle Chevalerie des ânes préparée par les Citoyens du Café Procope aux sacrés Infidèles à la Patrie, slnd (Ouzi Elyada, Presse populaire [...], op. cit., date ce pamphlet de novembre 1790).

(40) Je suis le véritable Père Duchesne, foutre!, $\mathrm{n}^{\circ} 78$ - septembre 1791.

(41) Annie Duprat, Les rois de papier. La caricature de Henri III à Louis XVI, Paris, Belin, 2002.

(42) Je suis le véritable Père Duchesne, foutre!, $\mathrm{n}^{\circ} 69$ - 1791.

(43) Ibid., $\mathrm{n}^{\mathrm{o}} 80-1791$. 
Que faire de tous ces conspirateurs, réels ou en puissance, dès lors que le roi des Français montre en personne l'exemple de la duplicité? Une fois de plus, journalistes et pamphlétaires s'en donnent à cœur joie pour tout à la fois faire rire les lecteurs et abattre leurs cibles par la dérision afin de mieux les réduire à l'impuissance. À l'instar du Carnaval, mais aussi des comédies qui plaisent au public communiant dans la théâtromanie tant dans les petits théâtres de la foire que sur les scènes les plus prestigieuses, la punition des "méchants" passe d'abord par coups de pied et autres coups de bâton. À tout seigneur tout honneur, le cul est omniprésent sitôt qu'un corps doit endurer un châtiment. Son premier intérêt, avant même de recevoir les coups, est de pouvoir être mis à nu en public, révélant ce qui doit être normalement caché avec le plus grand soin. Le monde à l'envers n'est pas loin, puisqu'il s'agit aussi de répliquer aux contrerévolutionnaires qui aiment à se gausser des sans-culottes en jouant sur le mot « cul ». Ainsi, dans « La Guerre constitutionnelle, poème héroi-lyripatrioti-burlesqui-comique » (tout un programme!), supposé chanter les exploits des «milices françaises » et de leur chef La Fayette, lorsqu'un "député de Paris » se présente au camp du général et que ce dernier demande des renseignements sur ce citoyen, on lui répond :

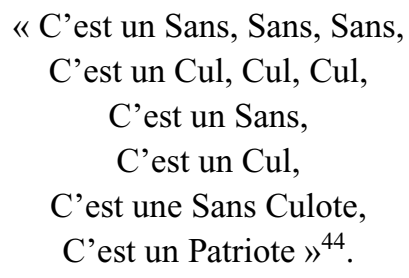

Quoi de mieux que de provoquer alors une énième inversion carnavalesque et de dépouiller ceux qui ne sortent jamais sans avoir « des culotes à leurs culs $[\ldots] \gg{ }^{45}$ ? Sitôt que le cri « culotte bas ! » retentit, tout « cul aristocrate » et tout arrière-train de clerc réfractaire doivent être « mis à l'air » ou « mis au vent », même si les contre-révolutionnaires s'empressent d'écrire avec humour que Dieu châtiera aussitôt quiconque osera dénuder le cul

(44) Les Sabbats Jacobites, $\mathrm{n}^{\circ}$ 73-74. Dans 1'affiche intitulée Qu'est-ce qu'un Sansculotte?, plus tardive (1792) et issue de la propagande girondine, l'association du cul au sans-culotte a disparu, preuve que nous sommes alors dans l'ordre de la réduction d'une exagération verbale, et non plus dans l'inversion burlesque.

(45) Grande découverte du Père Duchêne, sur la fausseté de la prétendue lettre des Princes [...] (Le Père Duchesne de Charles Fouilhoux, septembre 1791. Cf. Ouzi ElyadA, Presse populaire [...], op. cit., p. 150-152). 
d'un de ses serviteurs ${ }^{46}$. Les nonnes fessées par les patriotes, qui aiment à se délecter du spectacle ${ }^{47}$, ne parviennent pas ici à voler la vedette à l'abbé Maury, cible de prédilection des révolutionnaires et notamment du Père Duchesne d'Hébert. On ne compte plus les scénettes dans lesquelles le malheureux se retrouve le « cul à l'air », frappé à grands coups de pied ou fustigé avec divers instruments. En novembre 1790, sous le titre « Fais beau cul et tu n'en auras guères, ou l'abbé Maury fouetté par le Père Duchesne », une femme apporte au héros populaire « un bouillot tout neuf» (un bâton) et « [...] la correction commence », cependant qu'Hébert livre au ridicule la notion d'inviolabilité attachée à la personne des députés : " Il y aura peut-être quelque foutu bêtes qui regarderont mon action comme très punissable : comment, foutre, avoir mis au vent le cul d'un inviolable $»^{48}$ ! « Fais beau cul» se chantait peut-être sur un air connu, comme tant d'autres pièces de ce genre, et rappelait aussi les châtiments corporels infligés dans écoles et collèges. D'ailleurs, un autre pamphlet contemporain du précédent situe justement la même punition dans le cadre d'un collège, où l'abbé Maury a eu la mauvaise idée de se rendre pour visiter un professeur de ses amis. Hélas pour lui, il est reconnu par les écoliers.

« [...] Alors l'insurrection a été générale dans toutes les classes, et ces jeunes enfans, animés par le patriotisme, se sont écriés à l'aristocrate, et ne voulaient rien moins que le mettre à la lanterne. Les professeurs, alarmés du danger que courait l'abbé Maury, ont en vain employé les menaces, mais jamais Painblanc [c'est le nom du correcteur] n'a voulu se présenter avec les verges ».

Désormais assurés de ne pas encourir les verges redoutées s'ils s'en prennent à l'abbé, toutefois retenus par un soudain respect vis-à-vis de l'Assemblée constituante qui les pousse à écarter le supplice de la lanterne, les écoliers tiennent conseil - comme des grands, dans un monde à l'envers - et rendent le jugement suivant :

« L'abbé Maury déclaré atteint et convaincu d'être traître à la patrie, pour réparation de quoi est condamné à faire amende honorable aux portes

(46) Tel est le triste sort d'un aumônier jureur de Carcassonne chargé de fouetter quelques sœurs de la Charité coupables d'avoir refusé de paraître devant l'évêque constitutionnel : « Il allait procéder à l'exécution, quand tout à coup son corps devient noir comme un charbon, sa langue, de la même couleur, sort horriblement de sa bouche; il mord tous ceux qui l'approchent \& meurt dans cet horrible état » (La Rocambole des journaux, $\mathrm{n}^{\circ}$ 13, 12 février 1792). À force d'être " enragé », on le devient... et on ne porte pas impunément atteinte à des culs protégés de Dieu.

(47) Voir l'article d'Annie Duprat dans le présent numéro.

(48) Je suis le véritable Père Duchesne, foutre!, $\mathrm{n}^{\circ} 5$ - 1790. 
principales de la cour du Collège, culotte bas, où il recevra, de la main de Painblanc, trois coups de fouet. On observe qu'au moment de l'exécution, ledit abbé faisant des difficultés, Painblanc lui a dit : allons M. l'abbé, faites beau cul, vous n'aurez qu'une claque. Il s'y est soumis ${ }^{49}$.

La présence de cette «amende honorable » ne saurait tenir du hasard, puisque les individus condamnés par la justice royale étaient contraints sous l'Ancien Régime à une humiliation publique devant la justice de Dieu et des hommes avant de subir leur châtiment. Ici, le « cul mis au vent » sert à l'humiliation, qui plus est devant des enfants prompts à se moquer en pareille situation. Encore convient-il d'ajouter que l'abbé Maury s'en sort plutôt bien, dans la mesure où le « correcteur » se contente d'une « claque » sur ses parties charnues. En septembre 1791, une scène semblable se répète, mais cette fois Hébert entend faire rire des origines de « Maury-Crépin », l'abbé étant ainsi surnommé car son père, savetier de son état, est plusieurs fois mis en scène dans Le Père Duchesne. Au nom de saint Crépin, patron des cordonniers, un tire-pied de savetier manié avec vigueur assène des coups d'une tout autre nature qu'une simple claque : «Culotte bas, jean-foutre, ou la Grande Colère du père Duchesne qui a encore une fois foutu les étrivières à l'Abbé Maury, avec le tire-pied d'un Savetier du coin, pour le punir de tout le mal qu'il a fait à la Nation, \& de tous les complots qu'il médite encore $\wedge^{50}$.

Ces coups redoublés et ces culs meurtris suffisent-ils à arrêter lesdits complots? N'ont-ils pas, au contraire, pour effet de décupler la rage des $«$ aristocrates ${ }^{51}$ et ne convient-il pas dès lors de trouver une autre solution qu'un Carnaval hors saison ou un modeste charivari ${ }^{52}$,

(49) Faites beau cul, vous n'aurez qu'une claque, ou Événement arrivé à l'abbé Maury, dans la cour du Collège Mazarin, par les Ecoliers, le 27 à trois heures, Paris, Imprimerie de Chalon, rue du Théâtre Français, 1790.

(50) Je suis le véritable Père Duchesne, foutre!, $\mathrm{n}^{\circ} 79$ - 1791. Les étrivières (courroies servant à porter les étriers) peuvent parfois menacer de bien plus puissants que l'abbé Maury, si l'on en croit une confidence de Marie-Antoinette à son royal époux : «A voir le peuple vous mener, on dirait qu'il va vous crier : Haut le cul, les étrivières » (Anonyme, Le branle des Capucins, ou le Mille et Unième Tour de Marie-Antoinette. Petit Opéra Aristocratico-comico-risible en deux actes, SaintCloud, De l'imprimerie des clairvoyants, cul-de-sac des Recherches, 1791).

(51) C'est ce qu'affirme Lameth au cours d'un dîner avec Barnave et le héros populaire : «Père Duchêne, je pense comme vous, la rage des aristocrates est au comble, ils ont sur le cœur les coups de pieds au cul, ils écument comme des vipères blessés » (Le diné du Père Duchêne avec MM. de Lameth et Barnave [...]. Le Père Duchesne de la place Henri IV, de l'abbé Robin, $\mathrm{n}^{\circ} \mathrm{du} 15$ mars 1791. Cf. supra note 15).

(52) «Le premier jean-foutre qui s'avisera d'allarmer le public sera sur le champ happé... on le forcera à marcher à quatre pattes dans les rues; on lui attachera au cul toute l'édition de son ouvrage incendiaire » (à propos des journalistes et pamphlétaires contre-révolutionnaires. Je $m$ 'en $f \ldots$, ou Pensées de Jean Bart sur les affaires d'État, $\mathrm{n}^{\mathrm{o}} 20$, slnd [1790]). 
aussi drôles soient-ils? Le dentiste patriote qui arrache les dents trop longues de l' " aristocrate » et le perruquier patriote qui fait disparaître la tonsure d'un ci-devant moine à l'aide d'une perruque poudrée sont eux aussi assurés de faire rire ceux qui observent les caricatures, pour autant ramènent-ils à la raison les adversaires de la Révolution? Une fois de plus, le mot « cul » est convoqué sur le devant de la scène pour marquer le passage à une solution plus radicale : «foutre » tout ce petit monde dehors, d'abord des lieux où ils distillent leur « venin », ensuite du royaume. Les coups de pied s'imposent, comme il se doit, «[...] pour les foutre à la porte, les éconduire à coups de pied au cul $[\ldots]\rangle^{53}$, et de « culotte bas! » le slogan vengeur devient alors : « dispersez moi le reste à coup de pied au cul $[\ldots] »^{54}$. À tel point qu'il est permis d'ironiser sur une nouvelle armure que devraient adopter les chevaliers du régime défunt afin de protéger leur derrière, à défaut de leurs arrières (« Je suis sûr que beaucoup de ces gentils messieurs se feront foutre encore quelques coups de pied au cul. Gorsas, l'excellent Gorsas, leur recommande de se faire faire des culottes de fer-blanc $\rangle^{55}$ ). Une autre expression possède en la matière une vaste fortune et s'impose notamment de manière durable dans Le Père Duchesne d'Hébert : « foutre [ou donner, ou avoir] la pelle au cul ${ }^{56}$. Présente dans une Chanson patriotique du Père Duchesne sur le refus de la prestation du serment par les Évêques et les Curés ${ }^{57}$, ainsi que dans le titre d'une pièce de théâtre (L'Eau à la bouche, et la pelle au cul, et le déménagement \& le départ imprévu du Desservant intrus, histoire véritable ${ }^{58}$ ),

(53) Grande joie du Père Duchêne sur l'élargissement du curé d'Issy-l'évêque [...] (Le Père Duchesne de la place Henri IV, de l'abbé Robin, $\mathrm{n}^{\circ} \mathrm{du} 21$ mars 1791. Cf. supra note 15).

(54) Je suis le véritable Père Duchesne, foutre!, nº 118 - mars 1792.

(55) Soixantième lettre bougrement patriotiques du véritable Père Duchêne, de Lemaire, 24 mars 1791 (cf. supra note 16).

(56) En 1793 et 1794, le mot « cul » tend à se raréfier sous la plume d'Hébert, sauf dans cette expression encore assez présente $\left(n^{\circ} 265,287,332,333,337,342,355\right)$.

(57) « Pestant de se voir rasibus,

Et d'avoir tous la pelle au cul,

Y aisément cela se peut croire;

Ils voudraient ravoir leurs châteaux,

Leurs maîtresses et leurs chevaux,

Mais nom d'un chien!

On a l'moyen

D’leux casser à tous la mâchoire ». (Paris, Imprimerie de Chalon, l'an 2 de la liberté).

(58) Paris, Lallemand, 1791. Cette petite pièce anonyme raconte les vains espoirs d'un desservant qui aspire à devenir curé de sa paroisse, de laquelle les habitants le chassent manu militari. Il ambitionnait d'être curé, mais « après avoir eu l'eau à la bouche, il a fini la pelle au cul ». Et la pièce s'achève notamment par un petit couplet qui souligne un autre emploi du mot «cul » pour signifier un départ précipité : "L'intrus s'en va tout comme il est venu; sot comme une dinde il montre son cul $[\ldots] »$. 
elle autorise à son tour toute une liste de cibles désignées à la vindicte populaire, à l'exemple des officiers composant l'état-major de la garde nationale parisienne en juin 1792 : «Ses bons avis à la Garde nationale pour l'engager à foutre de la pêle au cul à l'état-major et à vivre en paix avec les braves sans-culottes $\gg{ }^{59}$. Et l'argument peut d'ailleurs se retourner, car si ces derniers restent sourds aux appels du Père Duchesne, ils risquent eux-mêmes de se faire « foutre la pelle au cul»:

«Certes, il n'est pas naturel

Que, dans son régime nouvel,

La France, en la main d'un seul homme,

Laisse une puissance, une somme,

Dont l'influence \& le calcul

Lui foutent de la pèle au cul $»^{60}$.

Donner ou recevoir « la pelle au cul »? Frapper ou être frappé? Certes, mais que se passera-t-il si les contre-révolutionnaires ne s'empressent pas de " foutre le camp » pour rejoindre les émigrés, comme s'ils avaient $«$ le feu au cul ${ }^{61}$ ? Le ton change alors et la menace se fait plus grave, même si le mot « cul » reste au centre du vocabulaire employé. Déjà en 1790, le titre explicite de l'anonyme passeport des Capucins, ou le Chapitre des coups de pied dans le cul, pour servir d'éclaircissement aux Aristocrates $^{62}$ annonçait les traditionnelles atteintes aux nobles et/ou religieux postérieurs, grâce auxquelles « [...] la noire cohorte s'est enfin dissipée $[\ldots]$ nos prélats antipatriotes, les Cazalès, les d'Esprémenil \& les abbés Maury, se sont enfoncés promptement dans leurs voitures, jusqu'à ce que leurs chevaux, plus prompts que les piétons, les aient entièrement dérobés aux yeux des spectateurs $\gg . .$. mais une ultime précision venait ponctuer cette fuite éperdue des Noirs. En effet, le pamphlet annonce que, s'ils se remettent à « cabaler», ils auront affaire à « [...] une compagnie patriotique, qui a un attachement inviolable à tous les arbres possibles, et notamment à tous les réverbères ». Et quand on observe à ses

(59) Je suis le véritable Père Duchesne, foutre!, nº 148 - 1792.

(60) Anonyme, Le Cantique séculaire du Père Duchesne, slnd. Un autre exemple est fourni par Le Père Duchesne à ses amis (1790) : «Mes bons amis, on se fout de nous, on voudrait la guerre civile, on voudrait nous désunir, parce qu'alors les Prussiens, les Autrichiens, les Pandours, les Hullans et mil autre Jean-foutres de cette espèce viendrait nous donner tout à leur aise de la pelle au cul, et puis au foutre la constitution ».

(61) «Vous croyez donc toujours que ça n'ira pas, sacrés mâtins que vous êtes, puisque vous foutez le camp, comme si vous aviez le feu au cul [...] » (Grande colère du Père Duchêne contre les nouveaux émigrans [...], Le Père Duchesne de Charles Fouilhoux, septembre 1791. Cf. note 45).

(62) Sl, Imprimerie de Malbouroux, 1790. 
membres qu'il n'est pas possible de faire justice en-dehors des lois, « [...] ils demandent quels sont les plus coupables, de ceux qui s'opposent au décret du drapeau rouge, ou de ceux qui s'opposent à tous les décrets de l'Assemblée [...] »? Avec cette allusion explicite à la loi martiale votée le 21 octobre 1789 et ce blanc-seing de facto donné à ceux qui, au nom de la légitimité, violeraient la légalité pour appliquer une justice "populaire » en lieu et place de la justice défaillante ${ }^{63}$, les culs ont tout intérêt à se protéger... Les contre-révolutionnaires ne quittent pas le royaume comme s'ils avaient « le feu au cul »? Qu'à cela ne tienne, il suffira de provoquer l'incendie, de leur « [... foutre] un barril de poudre au cul, si cela continue $»^{64}$, voire de les sodomiser avec des boulets pour être assuré du résultat $^{65}$ ! Les réduire à néant, les transformer en « torche-cul $»^{66}$, reviendrait somme toute à adopter des solutions dignes de tyrans fantasmés, mais ô combien définitives, ainsi que le suggèrent Jean Bart et son alter ego de papier lors d'une rencontre au cirque du Palais-Royal :

« Je voudrais qu'on adoptât en France un certain usage qui se pratiquait jadis en Asie. Quand un ministre mourait de sa belle mort, ou qu'on lui foutait la gueule en l'air, on couvrait de sa peau le siège de son successeur... - Ah! Duchesne mon ami, si l'on adoptait chez nous cette coutume, il faudrait un tanneur au cul des ministres... $\nu^{67}$.

(63) Même si journaux et pamphlets liés à l'univers du Père Duchesne sont souvent réticents à l'idée de passer à une violence plus grave et si lourde de conséquences : « [...] je crains qu'on fasse du hachis avec les aristocrates; et je voudrais qu'il fût possible de les convertir, plutôt que d'être obligé de les lanterner : ce qui arrivera; car on n'a d'abord corrigé leurs fureurs qu'avec des coups de pieds au cul, et je sens mon âme déchirée quand je songe qu'on n'aura plus pour eux aucun ménagement » (Soixante-dixième lettre bougrement patriotiques du véritable Père Duchêne, de Lemaire, 19 avril 1791. Cf. supra note 16). Sur les positions initiales d'Hébert à l'égard des violences populaires, voir Michel BIARD, « Des "bons avis" aux critiques assassines, la radicalisation d'Hébert mise en scène au fil des visites royales du Père Duchesne (décembre 1790 - décembre 1792) », $A H R F$, $\mathrm{n}^{\mathrm{o}} 3,2009$, p. 48-49.

(64) Sermon bougrement patriotique du véritable Père Duchesne, à l'occasion de la nouvelle Chevalerie des ânes préparée par les Citoyens du Café Procope aux sacrés Infidèles à la Patrie (cf. supra note 39).

(65) « [...] Je leur fous, le diable m'enlève, de la poudre \& des boulets dans le cul, jusqu'à ce qu'ils en aient tout leur saoul [...]" (Le Père Duchesne nommé commandant des troupes de ligne, que le roi envoye à Avignon [Le Père Duchesne de la rue du Vieux Colombier, 4-5 décembre 1790. Cf. Ouzi Elyada, Presse populaire [...], op. cit., p. 109-127]).

(66) «Aristocrate, tu m'indignes, foutre, je vais faire de toi un torche-cul à ma femme si tu n'abjure la contre-révolution » (Grand duel du Père Duchêne avec le sieur de Clermont-Tonnerre [...]. Le Père Duchesne de la place Henri IV, de l'abbé Robin, ${ }^{\circ}$ du 17 mars 1791. Cf. supra note $15)$.

(67) Je m'en $f \ldots$, ou Pensées de Jean Bart sur les affaires d'État, slnd [1790]. 
Les révolutionnaires héritent du XVIII ${ }^{\mathrm{e}}$ siècle trois genres comiques, le « bel esprit » des calotins, puis des persifleurs; le « langage populaire » de la farce grotesque inscrite dans la tradition burlesque; enfin, « la gaieté de l'esprit français ». Les conjuguent-ils uniquement dans une sorte de « gaieté nationale »? En suivant ici l'une de ces traditions, en adoptant le point de vue bakhtinien sur le burlesque et ses effets grotesques, avec son cortège de masques et d'inversions, avons-nous pris le risque de limiter descriptions et interprétations de la culture politique, qui plus est par le choix de la seule figure du cul? Précisons donc, en conclusion, la généralité de notre propos, son apport à la réflexion commune sur la culture de la Révolution française. Nous avons d'abord souligné la manière dont le Carnaval fait retour dans le contexte révolutionnaire de 1791, alors même qu'il est interdit par la municipalité de Paris, tandis qu'il se retrouve fictivement réalisé dans la production pamphlétaire. La voie ainsi ouverte par l'inversion burlesque, nous avons tenté de dégager, à partir de la description d'un corpus diversifié de textes imprimés de même facture et autour de la figure du cul, un moment révolutionnaire spécifique. En effet, s'y construit, par étapes, une part originale de la nouvelle culture politique dans le fait même de la diversification de ses objectifs, donc de son inscription dans la réalité politique en termes de contradiction, d'antagonisme, de conflit, de contestation, donc d'hétérogénéité. Il en ressort une description certes fortement réaliste (nous avons insisté sur ce point) et au plus près des antagonismes d'une société révolutionnaire, ce qui ne limite pas l'effet de réel à la représentation que cette société se donne d'elle-même dans un but unificateur. Nous sommes donc loin d'une politique culturelle homogénéisante qui serait voulue par les "élites» révolutionnaires. Il s'agit là plutôt d'un espace «médiat » aux modes d'appropriation politique particulièrement diversifiés, voire foisonnants ${ }^{68}$.

C'est dire aussi que les auteurs rencontrés dans cette brève enquête historico-textuelle ne cherchent pas à s'abaisser, le temps d'un divertissement, et tout particulièrement celui du Carnaval, au niveau d'un peuple supposé « vulgaire », du moins pour le milieu des patriotes. Ils écrivent dans un moment concret, le temps bien réel où se met peu à peu en place une pensée républicaine dans sa diversité même ${ }^{69}$, et pour ce faire ils ont recours à « une langue du peuple» immédiatement disponible dans sa

(68) On peut constater le même processus dans le cas de la chanson populaire (voir Laura Mason, Singing the French Revolution. Popular Culture and Politics, 1787-1799, Ithaca et Londres, Cornell University Press, 1996).

(69) Même si, bien sûr, quelques journalistes et pamphlétaires royalistes n'hésitent guère à employer, eux aussi, le mot « cul » et ses dérivés populaires. 
forme la plus usitée, la plus triviale, le burlesque. Nous sommes bien dans le registre des realia, exhibé ici dans la figure même du cul, de tradition esthétique fort ancienne. Ainsi, Jean-François Courouau ${ }^{70}$, dans son superbe ouvrage sur les écritures alternatives des langues minoritaires en France au début des temps modernes, précise, à propos des satires, que

« la présence des objets du réel trivial, des corps montrés dans toute leur matérialité, le foisonnement du concret dont tous ces textes portent la trace ostentatoire, signalent la volonté de faire parler le peuple, de lui faire dire, en dehors des filtres posés par la convention littéraire française - mais à l'intérieur des genres reconnus - quelque chose de sa propre réalité, en l'occurrence sa souffrance physique et morale ${ }^{71}$.

La figure du cul se prête particulièrement bien à cette façon de représenter le réel du quotidien populaire à travers les espoirs et les vicissitudes du peuple en révolution. Un genre mineur, en l'occurrence le burlesque et son cortège de grotesque, se trouve ainsi exhaussé, dans le fait même de la profusion de l'inventivité verbale, jusqu'à la production d'un lien sociopolitique dans le mouvement vers la matière de la vie, le quotidien et le réel, le trivial et le banal. Si le travail de la langue française n'est guère prédestiné jusqu'alors au burlesque, on peut se demander dans quelle mesure la Révolution n'ouvre pas une brèche dans la toute puissance d'un français issu d'un effort incessant de réduction analytique, via les grammaires et les dictionnaires, tout au long des temps modernes, grâce à une telle récréation d'une langue populaire à la fois inventive et politique.

Michel BIARD GRHis - Université de Rouen 61 rue Lord Kitchener 76600 Le Havre biard.m@wanadoo.fr

Jacques GuiLHAUMOU UMR « TRIANGLE », Université de Lyon, CNRS/ENS-LSH

15 Parvis Descartes 69342 Lyon CEDEX Jacques.Guilhaumou@univ-provence.fr

(70) Moun Lengatge Bèl. Les choix linguistiques minoritaires en France (1490-1660), Genève, Droz, 2008.

(71) Ibid.p. 150. 